\title{
Resolução espontânea de cisto aracnóideo suprasselar
}

\author{
Rodrigo Moreira Faleiro', Bárbara Faria Almada², \\ Patrícia Bernardes Silva3, Pedro Moreira Coelho Barroso², Isadora Maia Nobre ${ }^{2}$ \\ Hospital Felício Rocho, Belo Horizonte, MG, Brasil.
}

\section{RESUMO}

Cisto aracnóideo é uma coleção de líquido semelhante ao liquor, de parede formada por membranas aracnoides reforçadas por fibras colágenas. É uma anomalia congênita, benigna e rara, correspondendo a aproximadamente $1 \%$ das lesões expansivas intracranianas. Geralmente, os cistos aracnóideos (CAr) localizam-se na fossa média e, em apenas $10 \%$ dos casos, na região suprasselar. A involução espontânea dos CAr é um fato raro e pouco descrito na literatura. Relata-se um CAr suprasselar diagnosticado intraútero cuja conduta foi conservadora, observando-se sua involução espontânea à ressonância magnética de controle aos 24 meses de vida.

\section{PALAVRAS-CHAVE}

Cistos aracnóideos, remissão espontânea, imagem por ressonância magnética, cistos do sistema nervoso central.

\section{ABSTRACT}

Spontaneous resolution of a suprasellar arachnoid cyst

Arachnoid cyst is a benign fluid collection, formed by arachnoid bands. It is a rare congenital abnormality, representing approximately $1 \%$ of all intracranial expanding lesions. Generally, the arachnoid cysts (CAr) are located in the middle cranial fossa and only $10 \%$ occur in the suprasellar region. The spontaneous resolution of CAr is indeed a rare and poorly described evolution reported in the literature. We report a gestational diagnosed suprasellar cyst with spontaneous resolution at the 24th month old.

\section{KEYWORDS}

Arachnoid cysts, spontaneous remission, magnetic resonance imaging, central nervous system cysts.

\section{Introdução}

Cistos aracnóideos (CAr) são anomalias do desenvolvimento e correspondem a $1 \%$ das lesões expansivas intracranianas, sendo sua prevalência maior nas primeiras duas décadas de vida. ${ }^{1}$ Os CAr são envoltos por várias camadas de células da membrana aracnoide reforçadas por fibras colágenas e preenchidas por líquido semelhante ao liquor. ${ }^{1,2} \mathrm{~A}$ fossa média é o local mais comumente acometido e os cistos na região suprasselar constituem apenas $10 \%$ dos casos. ${ }^{3,4}$

O desenvolvimento e a história natural dos CAr são controversos. A maioria permanece estável e assintomática, podendo ser indicada conduta conservadora. Nos casos sintomáticos ou quando há crescimento, pode ser indicada cirurgia. ${ }^{5} \mathrm{Em}$ raros casos, pode ocorrer regressão do cisto, de forma espontânea ou associada a traumatismo craniano. ${ }^{6}$ Este trabalho relata um caso de resolução espontânea de CAr localizado na região suprasselar.

\section{Relato de caso}

Paciente HC, masculino, foi detectado com cisto intracraniano na ultrassonografia gestacional de rotina, realizada aos seis meses de gestação. Não havia hidrocefalia. À ressonância nuclear magnética (RNM) realizada aos oito meses de gestação, evidenciou-se imagem compatível com CAr suprasselar e, à RNM pós-nascimento, com 1 mês de idade, o cisto permanecia de tamanho inalterado e sem hidrocefalia associada (Figura 1).

1 Coordenador do serviço de Neurocirurgia e Neurologia do Hospital de Pronto-Socorro João XXIII, neurocirurgião do Hospital Felício Rocho, Belo Horizonte, MG, Brasil.

2 Acadêmico de Medicina da Universidade Federal de Minas Gerais (UFMG), Belo Horizonte, MG, Brasil.

3 Acadêmica de Medicina da Universidade José do Rosário Vellano, Belo Horizonte, MG, Brasil. 
Como o paciente encontrava-se assintomático e com exame de fundoscopia inalterado, foi indicada conduta conservadora, com realização de RNM a cada seis meses. Foram realizados quatro exames, aos 4, 10, 18 e 24 meses de vida. O desenvolvimento neuropsicomotor estava normal para a idade, bem como as medidas craniométricas. Aos 24 meses, sendo negada história de trauma ou sintomas neurológicos prévios, a RNM evidenciou regressão do cisto (Figura 2).
A

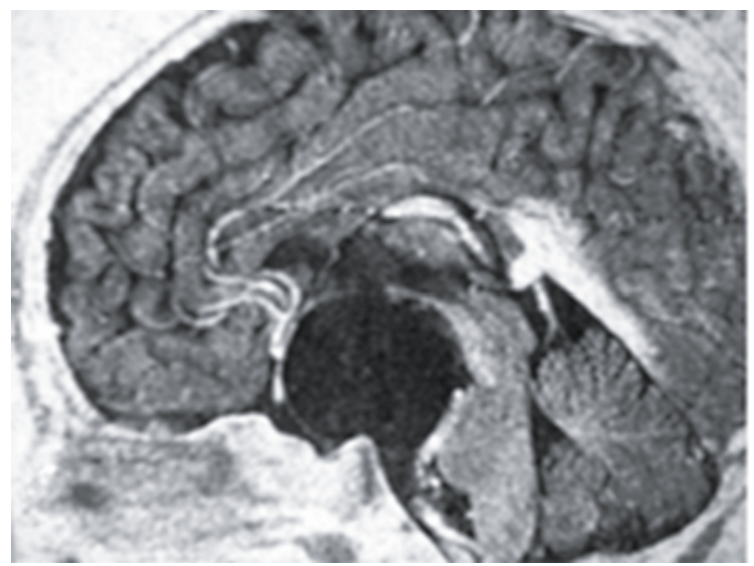

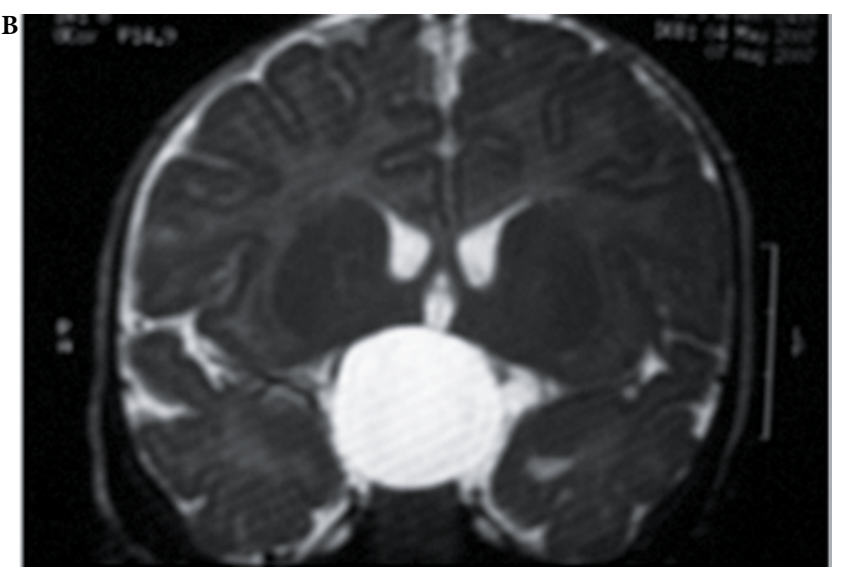

Figura 1 - RNM realizada com 1 mês de vida,ponderada em T1 (corte sagital em A) e T2 (corte coronal em B), evidencia lesão cística em região suprasselar, compatível com diagnóstico de cisto aracnóideo.
A

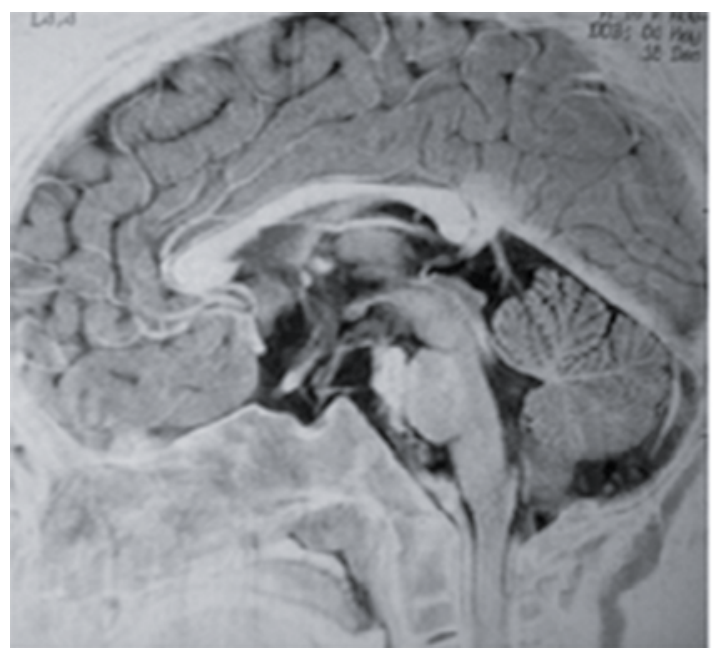

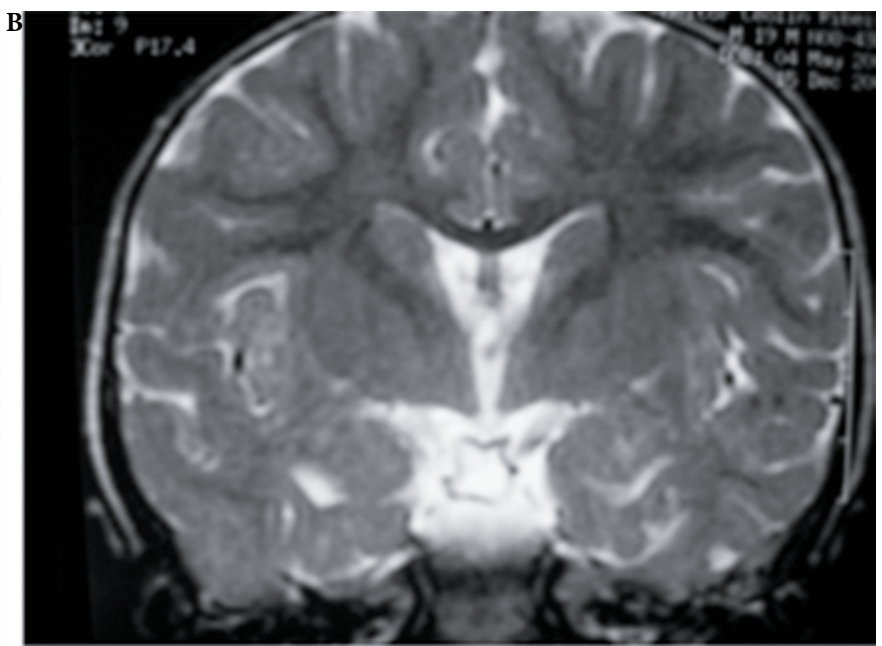

Figura 2 - RNM realizada aos 24 meses de idade, ponderada em T1 (corte sagital em A) e T2 (corte coronal em B), evidencia regressão do cisto.

\section{Discussão}

Acredita-se que a origem dos CAr deva-se a splitting ou duplicação da membrana aracnoide nos períodos iniciais de desenvolvimento embrionário. ${ }^{6} \mathrm{~A}$ formação de um mecanismo de válvula unidirecional ao redor da artéria basilar é postulado como responsável pela formação de cisto prepontino, que então cresceria e se estenderia à região suprasselar. ${ }^{4} \mathrm{~A}$ maior parte dos CAr permanece assintomática e, nesses casos, é indicado tratamento conservador, com realização periódica de exames de imagem para avaliar o crescimento da lesão., ${ }^{5,6}$ Os CAr podem causar sintomas à medida que crescem e exercem efeito de massa sobre estruturas adjacentes. ${ }^{7}$ Quando localizados na região suprasselar, podem comprimir o hipotálamo, quiasma óptico e vias de fluxo liquórico, causando cefaleias, vômitos, disfunções endócrinas e hidrocefalia. ${ }^{8}$ Nesses casos, é indicado tratamento cirúrgico, que inclui derivação cistoperitoneal, microcirurgia ou cistostomia endoscópica. ${ }^{1}$ 
No US pré-natal, os CAr aparecem como lesão hipoecoica, sendo a maioria diagnosticada no primeiro trimestre de gestação. O diagnóstico pré-natal de lesões hipoecoicas deve incluir o diagnóstico diferencial de CAr, cistos porencefálicos, cisto glioependimal, cisto do plexo coroide, aneurisma da veia de Galeno, esquizoencefalia, neoplasias e hemorragia intracraniana. ${ }^{8}$

O curso natural dos cistos aracnoides permanece indeterminado, havendo relatos de resolução espontânea. ${ }^{1,5,9}$ Uma possibilidade é que, após enfraquecimento focal da parede do cisto, ocorra comunicação entre o cisto e o espaço subdural, hipótese sustentada pelos relatos de regressão espontânea após traumatismo craniano leve. ${ }^{1,2,7}$ Acredita-se que, nesses casos, ocorra uma ruptura traumática do cisto e, com a resolução da coleção subdural, o cisto também desapareceria. Essa comunicação entre CAr e espaço subdural ocorre mais comumente nos cistos da fossa média. ${ }^{10}$

Uma segunda hipótese é a formação de comunicação entre o cisto e o espaço subaracnoide ou ventricular, possibilitando a drenagem do cisto por meio do fluxo normal do líquido cefalorraquidiano. ${ }^{1}$ Como a parede do cisto é mais espessa do que a membrana aracnoide normal, essa comunicação ocorreria com o rompimento onde a parede se encontra mais frágil. No caso de cistos suprasselares, a membrana ao redor da artéria basilar é o provável local de ocorrência. ${ }^{2} \mathrm{Na}$ ausência de traumatismo prévio, essa comunicação seria provocada por efeitos mecânicos sobre o tecido cerebral e o cisto, como os ocasionados por tosse, espirros ou prática de atividades físicas. ${ }^{6}$

A resolução espontânea de CAr é rara, e, dentre os 20 casos relatados na literatura, apenas dois eram localizados na região suprasselar. ${ }^{1,5,9}$ Entretanto, acredita-se que a ocorrência de CAr, bem como a resolução espontânea dessas anomalias, seja maior que a documentada, visto que muitos casos assintomáticos só são descobertos em exames de imagem realizados por outras indicações. Portanto, ao se considerar a intervenção cirúrgica para pacientes assintomáticos, deve-se considerar que a regressão espontânea, embora rara, é uma evolução possível.

\section{Referências}

1. Dodd RL, Barnes PD, Huhn SL. Spontaneous resolution of a prepontine arachnoid cyst. Case report and review of the literature. Pediatr Neurosurg. 2002;37(3):152-7.

2. Thomas BP, Pearson MM, Wushensky CA. Active spontaneous decompression of a suprasellar-prepontine arachnoid cyst detected with routine magnetic resonance imaging. Case report. J Neurosurg Pediatr. 2009;3(1):70-2.

3. Güzel A, Trippel M, Ostertage CB. Suprasellar arachnoid cyst: a 20-year follow-up after stereotactic internal drainage: case report and review of the literature. Turk Neurosurg. 2007;17(3):211-8.

4. Moon KS, Lee JK, Kim JH, Kim SH. Spontaneous disappearance of a suprasellar arachnoid cyst: case report and review of the literature. Childs Nerv Syst. 2007;23(1):99-104.

5. Seizeur R, Forlodou P, Coustans M, Dam-Hieu P. Spontaneous resolution of arachnoid cysts: review and features of an unusual case. Acta Neurochir (Wien). 2007;149(1):75-8.

6. Cincu R, Agrawal A, Eiras J. Intracranial arachnoid cysts: current concepts and treatment alternatives. Clin Neurol Neurosurg. 2007;109(10):837-43.

7. Briostol RE, Albuquerque FC, McDougal C, Spetzler RF. Arachnoid cysts: spontaneous resolution distinct from traumatic rupture. Case report. Neurosurg Focus, 2007;22(2):E2.

8. Chen CP. Prenatal diagnosis of arachnoid cysts. Taiwan J Obstet Gynecol. 2007;46(3):187-98.

9. Marlin E, Marlin A. Arachnoid cyst resolution. J Neurosurg Pediatr. 2010;5(3):310-1.

10. Inoue T, Matsushima T, Tashima S, Fukui M, Hasuo K. Spontaneous disappearance of a middle fossa arachnoid cyst associated with subdural hematoma. Surg Neurol. 1987;28(6):447-50.

Endereços para correspondência Rodrigo Moreira Faleiro

Rua Caraça, 518, ap. 201, Bairro Serra 30220-260 - Belo Horizonte, MG, Brasil

Telefone: (31) 9970-7676

E-mail: r.m.faleiro@hotmail.com

Bárbara Faria Almada

Rua Augustus Willian Parish, 15, Santa Amélia

31560-410 - Belo Horizonte, MG, Brasil

Telefone: (31) 9162-5031

E-mail: barbara.falmada@gmail.com 